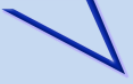

Free to Authors and Readers
DOAJ Seal

\title{
An eco-friendly approach for the synthesis of 1,2,5-trisubstituted and 4-amino- 1,2,5-tetrasubstituted imidazoles via a multi-component condensation
}

\author{
Hossein Mehrabi*, Farzaneh Alizadeh-Bami, Azam Meydani, and Soheila Besharat \\ Department of Chemistry, Vali-e-Asr University of Rafsanjan, 77176 Rafsanjan, Iran
}

E-mail:mehraby h@yahoo.com

Received 12-07-2020

Accepted 02-15-2020

Published on line $02-21-2021$

\section{Abstract}

The synthesis of 1,2,5-trisubstituted and 4-amino-1,2,5-tetrasubstituted imidazoles was demonstrated via a two-step cyclo-condensation reaction of aryl amines, carbonitriles, and ethyl bromopyruvate or aryl amines and arylglyoxals in ethanol heated under reflux in the presence of iron (III) chloride $\left(\mathrm{FeCl}_{3}\right)$, and disodium phosphate $\left(\mathrm{Na}_{2} \mathrm{HPO}_{4}\right)$ as catalysts, respectively. All the products were obtained in good to excellent yields and their structures were established from their spectroscopic data.

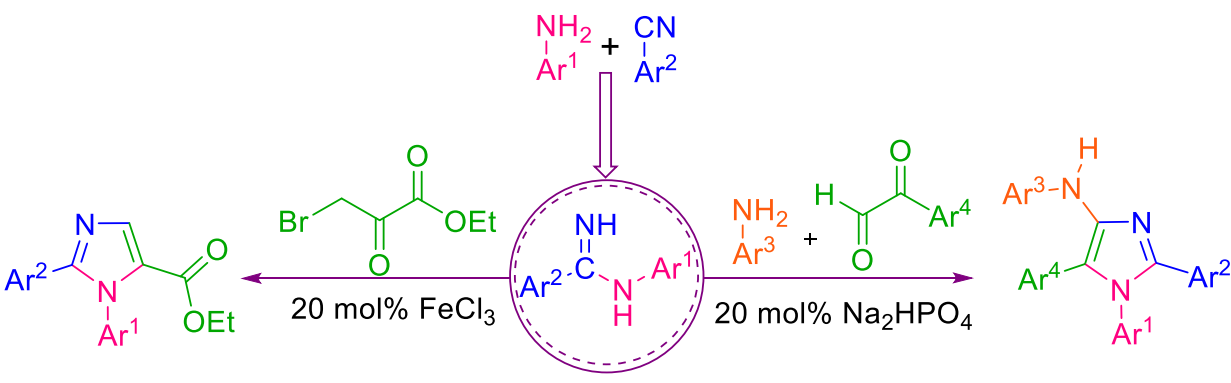

Keywords: Multi-component condensation, 1,2,5-trisubstituted imidazole, 4-amino-1,2,5-tetrasubstituted imidazole, arylglyoxal, ethyl bromopyruvate 


\section{Introduction}

Imidazoles are heterocyclic five-membered aromatic compounds with two 1,3 orientated nitrogen atoms, that have been of interesti to organic chemists owing to their useful biological activities, such as, antimicrobial, ${ }^{1}$ antitumor, ${ }^{2}$ antiviral, ${ }^{3}$ antifungal, ${ }^{4}$ and antioxidant activity. ${ }^{5}$ Moreover, the imidazole moiety exists in many natural products such as histidine, ${ }^{6}$ histamine, ${ }^{7}$ and biotin. ${ }^{8}$ Imidazoles also appear as the core structural skeleton in many important drugs such as miconazole, ${ }^{9}$ clotrimazole, ${ }^{10}$ econazole, ${ }^{11}$ losartan, ${ }^{12}$ and eprosartan. ${ }^{13}$ Importantly, imidazoles bearing an amino group at the 4-position have attracted significant attention because they are valuable synthons for the synthesis of biologically active molecules; 4aminoimidazoles have been used as the key intermediate for the synthesis of purine ${ }^{14,15}$ and guanidine. ${ }^{16}$ Therefore, 4-aminoimidazoles exhibit various biological applications such as antibacterial agents (I), ${ }^{1}$ anticancer agents (II), ${ }^{18}$ antidiabetic agents (III) ${ }^{19}$ kinase inhibitors (IV), ${ }^{20,21}$ and the treatment of Alzheimer's disease (V) (Figure 1). ${ }^{22}$ Due to the biological importance of imidazoles, the synthesis of polysubstituted imidazoles and 4-aminoimidazoles has attracted much attention in organic synthesis.

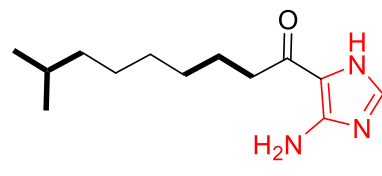

I (Antibacterial)

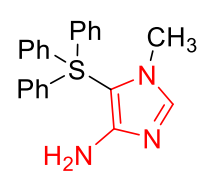

II (Anticancer)

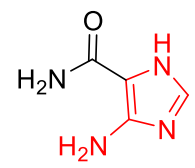

III (Antidiabetic)
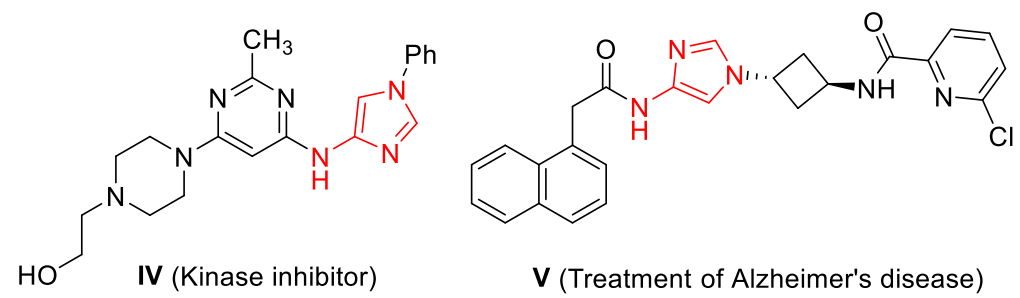

Figure 1. Selected drug molecules containing the 4-aminoimidazole moiety.

There are numerous reported syntheses of polysubstituted imidazoles and 4-aminoimidazoles, these include: (a) three or four component condensations of a 1,2-diketone, with an aldehyde, an amine or ammonium acetate by using various reagents or catalysts such as $\mathrm{CrCl}_{3} .6 \mathrm{H}_{2} \mathrm{O},{ }^{23} \mathrm{p}$-toluenesulfonic acid (PTSA), ${ }^{24}$ $\mathrm{CuCl}_{2} \cdot 2 \mathrm{H}_{2} \mathrm{O},{ }^{25} \mathrm{Fe}_{3} \mathrm{O}_{4} @ g-\mathrm{C}_{3} \mathrm{~N}_{4},{ }^{26} 3-\mathrm{N}$-morpholinopropanesulfonic acid (MOPS), ${ }^{27}$ silica sulfuric acid (SSA), ${ }^{28}$ ionic liquid, ${ }^{29}$ Caro's acid-silica gel, ${ }^{30}$ ultrasound irradiation; ${ }^{31}$ (b) $[3+2]$ cycloaddition; ${ }^{32,33}$ (c) via Ugi/Passerinireaction; ${ }^{34}$ (d) aza-Wittig reaction; ${ }^{35}$ and, (e) various other cyclization methods. ${ }^{36,37}$

However, the above-mentioned methods suffer from drawbacks such as tedious experimental procedures, low yields, use of expensive reagents, and complex workup and purification. Therefore, the development of more general eco-friendly procedures for the synthesis of polysubstituted imidazoles and specially 4-amino1,2,5-tetrasubstituted imidazoles from readily available starting materials remains an important task in organic chemistry.

With this in mind, and in continuation of our current studies on the development of new approaches for the synthesis of multi-substituted imidazoles, ${ }^{38-40}$ herein, we describe a facile and efficient protocol for the synthesis of 4-amino-1,2,5-tetrasubstituted and 1,2,5-trisubstituted imidazoles via a two-step, four or three- 
component reaction of aryl amines, carbonitriles, and aryl amines with arylglyoxals or ethyl bromopyruvate in the presence of disodium phosphate $\left(\mathrm{Na}_{2} \mathrm{HPO}_{4}\right)$, and iron (III) chloride $\left(\mathrm{FeCl}_{3}\right)$ as a catalyst, respectively.

\section{Results and Discussion}

To initiate our study, the arylamidine $\mathbf{3}$ was prepared via the reaction of aniline $\mathbf{1}$ with carbonitrile $\mathbf{2}$ in the presence of $\mathrm{AlCl}_{3}$ at $120^{\circ} \mathrm{C}$ for $1 \mathrm{~h}$ under solvent-free conditions. The arylamidine compounds were identified by the comparison of their physical and spectral data with those of authentic samples. ${ }^{41}$ Then, the optimised conditions for the condensation were established, using model compounds, arylamidine 3 ( $1 \mathrm{mmol})$, and electrophiles such as iminone resulting from aniline $4(1 \mathrm{mmol})$ with arylglyoxal 5 (1 $\mathrm{mmol})$ or ethyl bromopyruvate (7) $(1 \mathrm{mmol})$ in the presence of $\mathrm{Na}_{2} \mathrm{HPO}_{4}(20 \mathrm{~mol} \%)$, and $\mathrm{FeCl}_{3}(20 \mathrm{~mol} \%)$ as catalysts in ethanol heated at reflux for $12 \mathrm{~h}$. The optimized reaction conditions were then used to synthesize and explore the scope of this novel transformation to give two series of 4-amino-1,2,5-tetrasubstituted imidazoles 6, and 1,2,5-trisubstituted imidazoles $\mathbf{8}$. As can be seen from Table 1, the nature of the electrophilic component affects the type of products formed: when the electrophile is an iminone resulting from aniline 4 with arylglyoxal $\mathbf{5}$ then product $\mathbf{6}$ is formed, but when the electrophile is ethyl bromopyruvate (7) then product $\mathbf{8}$ is formed. Also, the catalysts affect the product yields: in the presence of both $\mathrm{Na}_{2} \mathrm{HPO}_{4}$ and $\mathrm{FeCl}_{3}$ as catalysts,

Table 1. Synthesis of 4-amino-1,2,5-tetrasubstituted imidazoles 6, and 1,2,5-trisubstituted imidazoles 8.

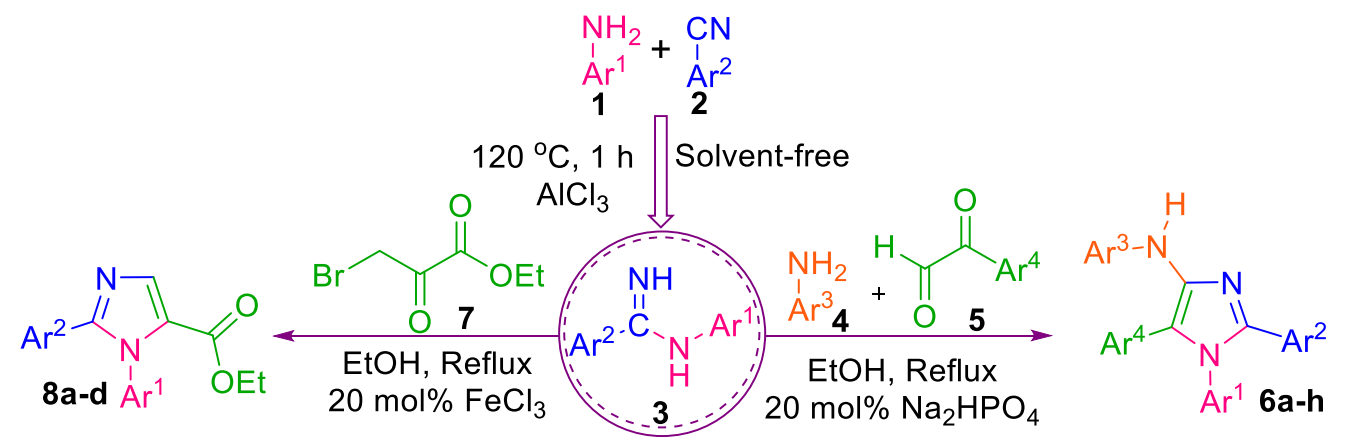

\begin{tabular}{|c|c|c|c|c|c|c|}
\hline Product & $A r^{1}$ & $A r^{2}$ & $A r^{3}$ & $A r^{4}$ & Catalyst & Yield $(\%)^{a, b}$ \\
\hline $6 a$ & $4-\mathrm{MeOC}_{6} \mathrm{H}_{4}$ & $\mathrm{Ph}$ & 4- $\mathrm{MeOC}_{6} \mathrm{H}_{4}$ & $4-\mathrm{ClC}_{6} \mathrm{H}_{4}$ & $\mathrm{Na}_{2} \mathrm{HPO}_{4}$ & 79 \\
\hline $6 b$ & $4-\mathrm{BrC}_{6} \mathrm{H}_{4}$ & $2-\mathrm{ClC}_{6} \mathrm{H}_{4}$ & $4-\mathrm{MeOC}_{6} \mathrm{H}_{4}$ & $4-\mathrm{ClC}_{6} \mathrm{H}_{4}$ & $\mathrm{Na}_{2} \mathrm{HPO}_{4}$ & 72 \\
\hline $6 c$ & $4-\mathrm{ClC}_{6} \mathrm{H}_{4}$ & $\mathrm{Ph}$ & 4-Tol & $4-\mathrm{ClC}_{6} \mathrm{H}_{4}$ & $\mathrm{Na}_{2} \mathrm{HPO}_{4}$ & 70 \\
\hline $6 d$ & $4-\mathrm{BrC}_{6} \mathrm{H}_{4}$ & $\mathrm{Ph}$ & 4-Tol & $4-\mathrm{ClC}_{6} \mathrm{H}_{4}$ & $\mathrm{Na}_{2} \mathrm{HPO}_{4}$ & 68 \\
\hline $6 e$ & 4-Tol & $2-\mathrm{ClC}_{6} \mathrm{H}_{4}$ & 4-Tol & $4-\mathrm{ClC}_{6} \mathrm{H}_{4}$ & $\mathrm{Na}_{2} \mathrm{HPO}_{4}$ & 80 \\
\hline $6 f$ & 4-Tol & $2-\mathrm{ClC}_{6} \mathrm{H}_{4}$ & $3-\mathrm{O}_{2} \mathrm{NC}_{6} \mathrm{H}_{4}$ & $4-\mathrm{ClC}_{6} \mathrm{H}_{4}$ & $\mathrm{Na}_{2} \mathrm{HPO}_{4}$ & 75 \\
\hline $6 g$ & $2,4-\mathrm{Me}_{2} \mathrm{C}_{6} \mathrm{H}_{3}$ & $\mathrm{Ph}$ & $3-\mathrm{O}_{2} \mathrm{NC}_{6} \mathrm{H}_{4}$ & $4-\mathrm{ClC}_{6} \mathrm{H}_{4}$ & $\mathrm{Na}_{2} \mathrm{HPO}_{4}$ & 74 \\
\hline $6 h$ & 4-Tol & $2-\mathrm{ClC}_{6} \mathrm{H}_{4}$ & 4- $\mathrm{MeOC}_{6} \mathrm{H}_{4}$ & $4-\mathrm{BrC}_{6} \mathrm{H}_{4}$ & $\mathrm{Na}_{2} \mathrm{HPO}_{4}$ & 82 \\
\hline $8 a$ & $\mathrm{Ph}$ & $2-\mathrm{ClC}_{6} \mathrm{H}_{4}$ & - & - & $\mathrm{FeCl}_{3}$ & 60 \\
\hline $8 b$ & 4- $\mathrm{BrC}_{6} \mathrm{H}_{4}$ & $2-\mathrm{ClC}_{6} \mathrm{H}_{4}$ & - & - & $\mathrm{FeCl}_{3}$ & 56 \\
\hline $8 c$ & $4-\mathrm{ClC}_{6} \mathrm{H}_{4}$ & $\mathrm{Ph}$ & - & - & $\mathrm{FeCl}_{3}$ & 60 \\
\hline $8 d$ & 4-Tol & $\mathrm{Ph}$ & - & - & $\mathrm{FeCl}_{3}$ & 62 \\
\hline
\end{tabular}

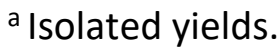

${ }^{b}$ Reaction time was $13 \mathrm{~h}$. 
a higher yield of the products $\mathbf{6}$ and $\mathbf{8}$ were obtained, respectively. Moreover, electronic effects and the nature of substituents on the aniline, the carbonitrile, and the arylglyoxal led to products with different reaction yields. When anilines containing electron-donating groups, were reacted with carbonitriles and the arylglyoxals containing electron-withdrawing groups, higher yields were obtained (Table 1).

To the best of our knowledge, all the synthesized compounds were new, as such, they were characterized by ${ }^{1} \mathrm{H}$ and ${ }^{13} \mathrm{C}$ NMR, IR, CHN analysis and melting points. For instance, the ${ }^{1} \mathrm{H}$ NMR spectrum of the compound 6a consisted of two singlets at $\delta_{\mathrm{H}} 3.59$ and 3.73 for the methoxy groups in the product. The aromatic protons resonated in the region $\delta_{\mathrm{H}}$ 6.45-7.91 and a broad singlet signal at $\delta_{\mathrm{H}} 7.44$ for the proton of the nitrogen group were also observed. The ${ }^{13} \mathrm{C}$ NMR spectrum of compound 6a exhibited 21 distinct signals in agreement with the proposed structure. In the IR spectrum, the $\mathrm{NH}$ and $\mathrm{C}=\mathrm{N}$ groups absorption were observed at 3409 and $1608 \mathrm{~cm}^{-1}$. Partial assignments of these resonances for the other products are given in the experimental section.

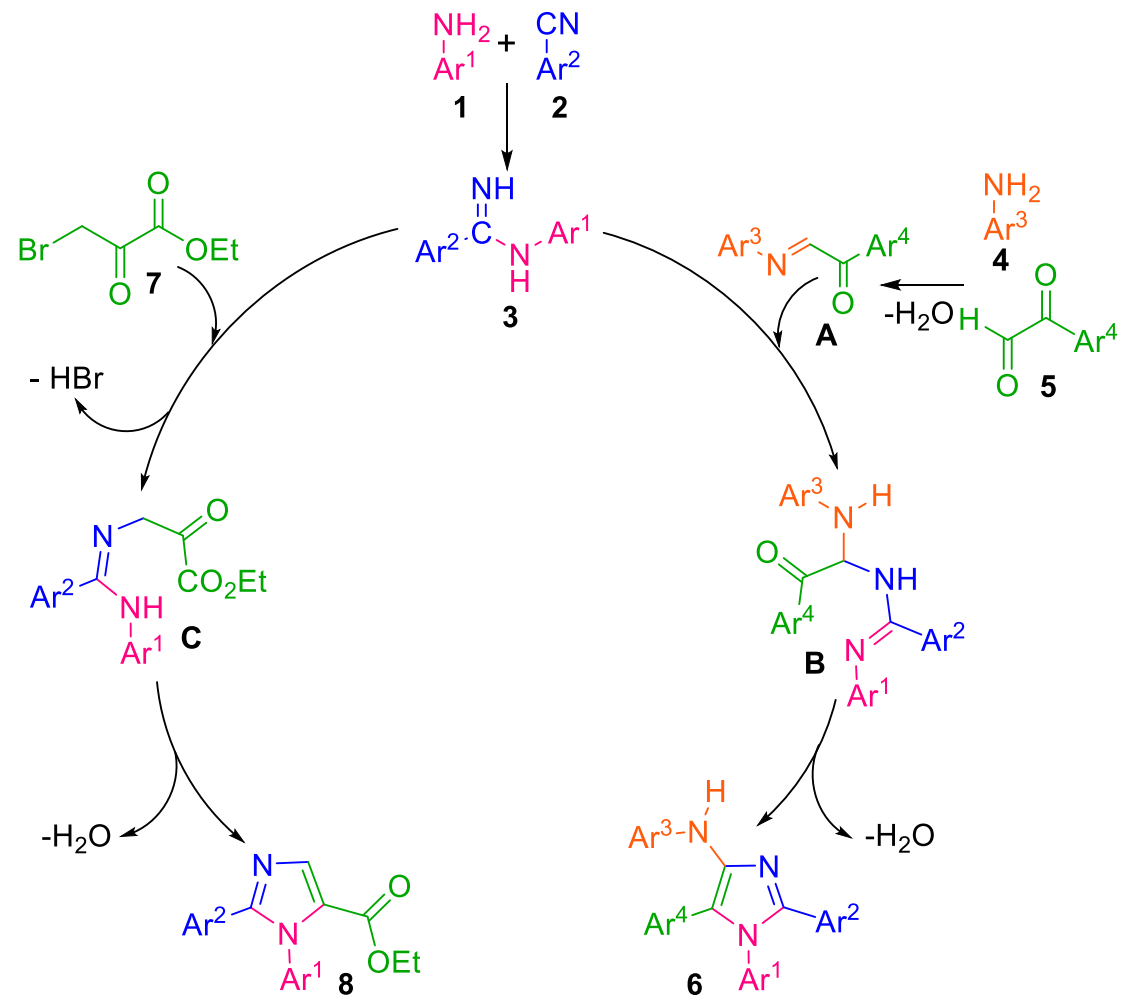

Scheme 1. Proposed mechanism for the synthesis of 4-amino-1,2,5-tetrasubstituted imidazoles 6, and 1,2,5trisubstituted imidazoles 8.

A proposed mechanism for the formation of 4-amino-1,2,5-tetrasubstituted imidazoles 6 and 1,2,5trisubstituted imidazoles $\mathbf{8}$ is described in Scheme 1. The nature of the electrophile was different, so that: 4amino-1,2,5-tetrasubstituted imidazoles 6 formed via four steps; at first, based on the result in the current study and our previous report, ${ }^{40}$ arylamidine $\mathbf{3}$ is formed by the addition of the aniline $\mathbf{1}$ to the carbonitrile $\mathbf{2}$ at $120{ }^{\circ} \mathrm{C}$ for $1 \mathrm{~h}$ under solvent-free conditions. Also, iminone $\mathbf{A}$ is formed through Knoevenagel condensation of the aniline $\mathbf{4}$ to the arylglyoxal 5, in agreement with our previous report. ${ }^{42}$ Then, on addition of arylamidine $\mathbf{3}$, the formation of intermediate $\mathbf{B}$ occurred via intermolecular nucleophilic addition of the nitrogen at the imine carbon atom of $\mathbf{A}$, which subsequently undergoes intramolecular nucleophilic addition the second nitrogen to the carbonyl group and elimination of $\mathrm{H}_{2} \mathrm{O}$ affording the 4-amino-1,2,5-tetrasubstituted imidazole 6. 1,2,5- 
Trisubstituted imidazole $\mathbf{8}$ is formed, according to the mechanism of the previous works. ${ }^{43-45}$ Firstly, arylamidine $\mathbf{3}$ is formed by the addition of the aniline 1 to the carbonitrile $\mathbf{2}$ at $120^{\circ} \mathrm{C}$ for $1 \mathrm{~h}$ under solventfree conditions. Then, on addition of arylamidine $\mathbf{3}$, the formation of intermediate $\mathbf{C}$ occurred via a nucleophilic addition reaction of the nitrogen to methylene group in ethyl bromopyruvate (7) followed by the elimination of $\mathrm{HBr}$. In the last step, intermediate $\mathbf{C}$ undergoes intramolecular nucleophilic addition the second nitrogen to the carbonyl group and elimination of $\mathrm{H}_{2} \mathrm{O}$ to form the 1,2,5-trisubstituted imidazole 8 .

\section{Conclusions}

In summary, we have successfully described a convenient and efficient protocol for the synthesis of two series of 4-amino-1,2,5-tetrasubstituted and 1,2,5-trisubstituted imidazoles via a two-step, four or three-component reaction of aryl amines, carbonitriles, and aryl amines with arylglyoxals or ethyl bromopyruvate in the presence of disodium phosphate, and iron (III) chloride as catalyst, respectively. The catalysts are readily available and inexpensive and can conveniently be handled and removed from the reaction mixture. The notable features offered by this method are the environmentally friendly procedure, readily available starting materials, easy workup, and good to high product yields.

\section{Experimental Section}

General. All chemicals were purchased from Aldrich and Merck with high-grade quality, and used without any purification. All melting points were obtained by Barnstead Electrothermal 9200 apparatus and are uncorrected. The reactions were monitored by TLC and all yields refer to isolated products. NMR spectra were obtained on a Varian $500 \mathrm{MHz}$ spectrometer $\left({ }^{1} \mathrm{H} \mathrm{NMR}\right.$ at $500 \mathrm{MHz},{ }^{13} \mathrm{C} \mathrm{NMR}$ at $\left.125 \mathrm{MHz}\right)$ using TMS as an internal standard; coupling constants are measured in $\mathrm{Hz}$. Infrared spectra were recorded on a Bruker FT-IR Equinax-55 spectrophotometer in $\mathrm{KBr}$ with absorption in $\mathrm{cm}^{-1}$. Elemental analyses were performed using a Carlo Erba EA 1108 instrument. All products were characterized by their spectral and physical data.

General procedure for the synthesis of arylamidine (3). The mixture of aryl amine 1 (1.2 mmol), carbonitrile 2 $(1.0 \mathrm{mmol})$, and $\mathrm{AlCl}_{3}(1.0 \mathrm{mmol})$ was charged to a round bottom flask. The mixture was stirred at $120{ }^{\circ} \mathrm{C}$ for 1 $\mathrm{h}$, then ice water $(20 \mathrm{~mL})$ was added to round bottom flask heat mixture, after $10 \%$ sodium hydroxide solution was added with a mixture of reaction to obtain $\mathrm{pH}=14$, and the precipitate was extracted with $\mathrm{CHCl}_{3}(3 \times 7$ $\mathrm{mL})$, dried $\left(\mathrm{MgSO}_{4}\right)$, and the solvent evaporated. The colorless crude product was crystallized from toluene.

General procedure for the synthesis of compounds $6 \mathrm{a}-\mathrm{h}$. A mixture of arylamine 4 (1.0 mmol) and arylglyoxal $5(1.0 \mathrm{mmol})$ was stirred in $\mathrm{EtOH}(15 \mathrm{~mL})$ heated at reflux for $2 \mathrm{~h}$ to give iminone. Then, arylamidine 3 (1.0 $\mathrm{mmol})$ was added in the presence of disodium phosphate $(20 \mathrm{~mol} \%)$ under reflux conditions for $10 \mathrm{~h}$. After completion of the reaction (TLC), the solvent was removed under reduced pressure, and the resulting crude product was purified by washing with $\mathrm{EtOH}$ to give the pure compounds $6 \mathbf{a}-\mathbf{h}(68-82 \%)$.

General procedure for the synthesis of compounds 8a-d. A mixture of arylamidine 3 (1.0 mmol) and ethyl bromopyruvate (7) $(1.0 \mathrm{mmol})$ was stirred in $\mathrm{EtOH}(15 \mathrm{~mL})$ in the presence of iron (III) chloride (20 mol\%) heated at reflux for $12 \mathrm{~h}$. After completion of the reaction (TLC), the solvent was removed under reduced pressure, and the viscous residue was purified by plate chromatography $(20 \times 20 \mathrm{~cm})$ using $n$-hexane/EtOAc $(25: 75)$ as eluent to give the pure compounds $8 a-d(56-62 \%)$. 
5-(4-Chlorophenyl)-N,1-bis(4-methoxyphenyl)-2-phenyl-1H-imidazol-4-amine (6a). Colorless solid; $\mathrm{mp:} \mathrm{118-}$ $120{ }^{\circ} \mathrm{C}$. IR v/cm ${ }^{-1}(\mathrm{KBr}): 3409(\mathrm{NH}), 1608(\mathrm{C}=\mathrm{N}) \mathrm{cm}^{-1} .{ }^{1} \mathrm{H}$ NMR (500 MHz, DMSO-d6): $\delta 3.59\left(\mathrm{~s}, 3 \mathrm{H}, \mathrm{OCH}_{3}\right), 3.73$ $\left(\mathrm{s}, 3 \mathrm{H}, \mathrm{OCH}_{3}\right), 6.45$ (d, J 9.0, 2H, ArH), 6.64 (d, J 9.0, 2H, ArH), 6.91 (d, J 9.0, 2H, ArH), 7.13-7.38 (m, 9H, ArH), 7.44 (bs, $1 \mathrm{H}, \mathrm{NH}), 7.91$ (d, J 9.0, 2H, ArH). ${ }^{13} \mathrm{C}$ NMR (125 MHz, DMSO-d6): $\delta_{\mathrm{c}} 55.5,55.7,114.7,115.1,127.1$, 128.3, 128.6, 128.6, 128.6, 128.7, 129.3, 129.5, 129.9, 131.0, 131.1, 140.4, 144.2, 152.3, 156.7, $159.5,161.1$. Anal. Calcd for $\mathrm{C}_{29} \mathrm{H}_{24} \mathrm{ClN}_{3} \mathrm{O}_{2}$ (481.98): C, 72.27; $\mathrm{H}, 5.02 ; \mathrm{N}, 8.72$. Found: $\mathrm{C}, 72.53 ; \mathrm{H}, 5.07 ; \mathrm{N}, 8.65$.

1-(4-Bromophenyl)-2-(2-chlorophenyl)-5-(4-chlorophenyl)-N-(4-methoxyphenyl)-1H-imidazol-4-amine (6b). Colorless solid; mp: $116-118{ }^{\circ} \mathrm{C}$. IR v/cm ${ }^{-1}(\mathrm{KBr}): 3340(\mathrm{NH}), 1614(\mathrm{C}=\mathrm{N}) \mathrm{cm}^{-1}$. ${ }^{1} \mathrm{H}$ NMR $\left(500 \mathrm{MHz}, \mathrm{DMSO}-d_{6}\right): \delta_{\mathrm{H}}$ $3.81\left(\mathrm{~s}, 3 \mathrm{H}, \mathrm{OCH}_{3}\right), 6.53-6.58(\mathrm{~m}, 3 \mathrm{H}, \mathrm{ArH}), 6.91(\mathrm{~d}, 8.5,2 \mathrm{H}, \mathrm{ArH}), 7.06(\mathrm{~d}, J$ 8.5, 2H, ArH), 7.51-7.64 (m, 7H, $\operatorname{ArH}), 8.20(\mathrm{~d}, J$ 8.5, $2 \mathrm{H}, \mathrm{ArH}), 8.47$ (bs, $1 \mathrm{H}, \mathrm{NH}) .{ }^{13} \mathrm{C} \mathrm{NMR}\left(125 \mathrm{MHz}, \mathrm{DMSO}-d_{6}\right): \delta_{\mathrm{C}} 55.9,115.1,124.4,125.7$, 127.0, 127.9, 128.3, 128.6, 129.0, 129.3, 130.0, 130.6, 131.1, 131.4, 131.7, 132.5, 134.5, 138.8, 140.9, 141.5, 154.8, 160.6. Anal. Calcd for $\mathrm{C}_{28} \mathrm{H}_{20} \mathrm{BrCl}_{2} \mathrm{~N}_{3} \mathrm{O}$ (565.29): $\mathrm{C}, 59.49 ; \mathrm{H}, 3.57 ; \mathrm{N}, 7.43$. Found: $\mathrm{C}, 59.81 ; \mathrm{H}, 3.61 ; \mathrm{N}$, 7.26 .

1,5-Bis(4-chlorophenyl)-2-phenyl- $\mathrm{N}$-(p-tolyl)-1H-imidazol-4-amine (6c). Colorless solid; mp: $122-124{ }^{\circ} \mathrm{C}$. IR v/cm ${ }^{-1}(\mathrm{KBr}): 3409(\mathrm{NH}), 1617(\mathrm{C}=\mathrm{N}) \mathrm{cm}^{-1} .{ }^{1} \mathrm{H}$ NMR (500 MHz, DMSO-d $\left.d_{6}\right): \delta_{\mathrm{H}} 2.28\left(\mathrm{~s}, 3 \mathrm{H}, \mathrm{CH}_{3}\right), 6.33(\mathrm{~d}, \mathrm{~J} 8.5,1 \mathrm{H}$, ArH), $6.43(\mathrm{~d}, J 8.5,2 \mathrm{H}, \mathrm{ArH}), 6.75(\mathrm{~d}, J 8.5,2 \mathrm{H}, \mathrm{ArH}), 6.83(\mathrm{~d}, J 8.5,2 \mathrm{H}, \mathrm{ArH}), 7.01$ (d, J 8.5, 2H, ArH), 7.15-7.22 $(\mathrm{m}, 4 \mathrm{H}, \mathrm{ArH}), 7.53$ (d, J 8.5, 2H, ArH), 7.70 (bs, $1 \mathrm{H}, \mathrm{NH}), 7.92$ (d, J 8.5, 2H). ${ }^{13} \mathrm{C} \mathrm{NMR}\left(125 \mathrm{MHz}, \mathrm{DMSO}-d_{6}\right): \delta_{\mathrm{c}}$ 21.4, 113.4, 122.2, 125.7, 127.3, 127.8, 128.4, 128.5, 128.6, 128.7, 128.7, 129.1, 129.2, 129.3, 129.6, 130.1, 140.5, 144.1, 144.3, 162.9. Anal. Calcd for $\mathrm{C}_{28} \mathrm{H}_{21} \mathrm{Cl}_{2} \mathrm{~N}_{3}$ (470.40): C, 71.49; $\mathrm{H}, 4.50 ; \mathrm{N}$, 8.93. Found: $\mathrm{C}, 71.13 ; \mathrm{H}$, $4.46 ; \mathrm{N}, 8.86$.

1-(4-Bromophenyl)-5-(4-chlorophenyl)-2-phenyl- $\mathbf{N}$-(p-tolyl)-1H-imidazol-4-amine (6d). Colorless solid; $\mathrm{mp}$ : 127-129 ${ }^{\circ} \mathrm{C}$. IR v/cm ${ }^{-1}(\mathrm{KBr}): 3412(\mathrm{NH}), 1617(\mathrm{C}=\mathrm{N}) \mathrm{cm}^{-1} .{ }^{1} \mathrm{H}$ NMR $\left(500 \mathrm{MHz}, \mathrm{DMSO}-d_{6}\right): \delta_{\mathrm{H}} 2.29\left(\mathrm{~s}, 3 \mathrm{H}, \mathrm{CH}_{3}\right)$, $6.43(\mathrm{~d}, J$ 8.5, 2H, ArH), $6.84(\mathrm{~d}, J$ 8.5, 2H, ArH), 7.15-7.39 (m, 9H, ArH), 7.59 (d, J 9.0, 2H, ArH), 7.70 (bs, 1H, $\mathrm{NH}), 7.91$ (d, J 9.0, 2H, ArH). ${ }^{13} \mathrm{C}$ NMR (125 MHz, DMSO- $\left.d_{6}\right): \delta_{\mathrm{C}} 20.5,113.3,122.2,125.7,127.3,128.4,128.6$, 128.6, 128.7, 128.8, 128.9, 129.1, 129.3, 130.1, 130.4, 132.5, 140.3, 144.1, 144.3, 158.4. Anal. Calcd for $\mathrm{C}_{28} \mathrm{H}_{21} \mathrm{BrClN}_{3}$ (514.85): C, 65.32; $\mathrm{H}, 4.11 ; \mathrm{N}, 8.16$. Found: $\mathrm{C}, 65.50 ; \mathrm{H}, 4.15 ; \mathrm{N}, 8.03$.

2-(2-Chlorophenyl)-5-(4-chlorophenyl)-N,1-di-p-tolyl-1H-imidazol-4-amine (6e). Colorless solid; mp: $130-132$ ${ }^{\circ} \mathrm{C}$. IR v/cm ${ }^{-1}(\mathrm{KBr}): 3414(\mathrm{NH}), 1613(\mathrm{C}=\mathrm{N}) \mathrm{cm}^{-1} .{ }^{1} \mathrm{H}$ NMR $\left(500 \mathrm{MHz}, \mathrm{DMSO}-d_{6}\right): \delta_{\mathrm{H}} 2.06\left(\mathrm{~s}, 3 \mathrm{H}, \mathrm{CH}_{3}\right), 2.11(\mathrm{~s}, 3 \mathrm{H}$, $\left.\mathrm{CH}_{3}\right), 6.42(\mathrm{~d}, J$ 9.0, $1 \mathrm{H}, \mathrm{ArH}), 6.70-6.78(\mathrm{~m}, 3 \mathrm{H}, \mathrm{ArH}), 6.87-6.91(\mathrm{~m}, 4 \mathrm{H}, \mathrm{ArH}), 7.25(\mathrm{~d}, J$ 9.0, $1 \mathrm{H}, \mathrm{ArH}), 7.36-7.50$ $(\mathrm{m}, 5 \mathrm{H}, \mathrm{ArH}), 7.50$ (bs, $1 \mathrm{H}, \mathrm{NH}), 7.58$ (d, J 8.5, 2H, ArH). ${ }^{13} \mathrm{C}$ NMR (125 MHz, DMSO-d $)$ : $\delta_{\mathrm{c}} 20.4,20.8,113.4$, $113.7,122.2,125.7,126.3,127.2,127.4,127.8,128.3,129.4,129.6,129.9,130.4,131.4,131.5,132.3,132.5$, $144.8,146.0,156.8,161.3$.

2-(2-Chlorophenyl)-5-(4-chlorophenyl)- $\mathbf{N}$-(3-nitrophenyl)-1-(p-tolyl)-1H-imidazol-4-amine (6f). Colorless solid; mp: $142-144{ }^{\circ} \mathrm{C}$. IR v/cm ${ }^{-1}(\mathrm{KBr}): 3403(\mathrm{NH}), 1619(\mathrm{C}=\mathrm{N}) \mathrm{cm}^{-1} .{ }^{1} \mathrm{H}$ NMR $\left(500 \mathrm{MHz}, \mathrm{DMSO}-d_{6}\right): \delta_{\mathrm{H}} 2.10(\mathrm{~s}$, $\left.3 \mathrm{H}, \mathrm{CH}_{3}\right), 6.87-6.95(\mathrm{~m}, 6 \mathrm{H}, \mathrm{ArH}), 7.22(\mathrm{~d}, J$ 8.5, 2H, ArH), 7.28 (bs, 1H, NH), 7.40-7.50 (m, 5H, ArH), $7.63(\mathrm{~d}, J$ 8.5, $2 \mathrm{H}, \mathrm{ArH}), 8.11(\mathrm{~d}, J \mathrm{9} .0,1 \mathrm{H}, \mathrm{ArH}) .{ }^{13} \mathrm{C}$ NMR $\left(125 \mathrm{MHz}, \mathrm{DMSO}-d_{6}\right): \delta_{\mathrm{c}} 20.8,112.2,120.8,125.8,126.5,127.2$, $127.5,127.9,128.4,129.2,129.3,129.5,129.6,130.0,131.5,131.6,131.6,132.5,135.4,135.5,136.5,141.0$, 148.5, 161.4. Anal. Calcd for $\mathrm{C}_{28} \mathrm{H}_{20} \mathrm{Cl}_{2} \mathrm{~N}_{4} \mathrm{O}_{2}$ (515.39): $\mathrm{C}, 65.25 ; \mathrm{H}, 3.91 ; \mathrm{N}, 10.87$. Found: $\mathrm{C}, 65.41 ; \mathrm{H}, 3.97 ; \mathrm{N}$, 10.65 .

5-(4-Chlorophenyl)-1-(2,4-dimethylphenyl)-N-(3-nitrophenyl)-2-phenyl-1H-imidazol-4-amine (6g). Colorless solid; mp: $146-148{ }^{\circ} \mathrm{C}$. IR v/cm ${ }^{-1}(\mathrm{KBr}): 3414(\mathrm{NH}), 1620(\mathrm{C}=\mathrm{N}) \mathrm{cm}^{-1} .{ }^{1} \mathrm{H}$ NMR $\left(500 \mathrm{MHz}, \mathrm{DMSO}-d_{6}\right): \delta_{\mathrm{H}} 2.06(\mathrm{~s}$, $\left.3 \mathrm{H}, \mathrm{CH}_{3}\right), 2.18\left(\mathrm{~s}, 3 \mathrm{H}, \mathrm{CH}_{3}\right), 6.78-6.95(\mathrm{~m}, 4 \mathrm{H}, \mathrm{ArH}), 7.22-7.32(\mathrm{~m}, 5 \mathrm{H}, \mathrm{ArH}), 7.45-7.59(\mathrm{~m}, 5 \mathrm{H}, \mathrm{ArH}, \mathrm{NH}), 7.68(\mathrm{~d}$, J 8.5, $2 \mathrm{H}, \mathrm{ArH}), 8.11$ (d, J 8.5, $1 \mathrm{H}, \mathrm{ArH}) .{ }^{13} \mathrm{C}$ NMR (125 MHz, DMSO- $\left.d_{6}\right): \delta_{\mathrm{c}} 20.8,20.9,119.6,119.7$ 120.3, 127.5, 
127.7, 128.3, 128.4, 128.7, 129.0, 129.2, 129.4, 130.6, 130.8, 131.5, 131.9, 131.9, 132.5, 143.1, 148.3, 148.4, 148.7, 149.1, 162.3. Anal. Calcd for $\mathrm{C}_{29} \mathrm{H}_{23} \mathrm{ClN}_{4} \mathrm{O}_{2}$ (494.98): C, 70.37; $\mathrm{H}, 4.68 ; \mathrm{N}, 11.32$. Found: $\mathrm{C}, 70.32 ; \mathrm{H}, 4.60$; N, 11.19.

5-(4-Bromophenyl)-2-(2-chlorophenyl)-N-(4-methoxyphenyl)-1-(p-tolyl)-1H-imidazol-4-amine (6h). Colorless solid; mp: $125-127^{\circ} \mathrm{C}$. IR v/cm ${ }^{-1}(\mathrm{KBr}): 3377(\mathrm{NH}), 1606(\mathrm{C}=\mathrm{N}) \mathrm{cm}^{-1} .{ }^{1} \mathrm{H}$ NMR $\left(500 \mathrm{MHz}, \mathrm{DMSO}-d_{6}\right): \delta_{\mathrm{H}} 2.11(\mathrm{~s}$, $\left.3 \mathrm{H}, \mathrm{CH}_{3}\right), 3.56\left(\mathrm{~s}, 3 \mathrm{H}, \mathrm{OCH}_{3}\right), 6.46(\mathrm{~d}, J$ 8.5, 2H, ArH), $6.55(\mathrm{t}, J$ 8.5, 2H, ArH), 6.84-6.89 (m, 4H, ArH), $7.04(\mathrm{~d}, J$ 8.5, 1H, ArH), $7.25(\mathrm{t}, J 8.5,1 \mathrm{H}, \mathrm{ArH}), 7.40(\mathrm{~d}, J$ 8.5, 2H, ArH), $7.56(\mathrm{~d}, J$ 8.5, 2H, ArH), $7.62(\mathrm{t}, J$ 8.5, 1H, ArH), $8.19(\mathrm{~d}, J 8.5,1 \mathrm{H}, \mathrm{ArH}), 8.46$ (bs, $1 \mathrm{H}, \mathrm{NH}) .{ }^{13} \mathrm{C}$ NMR $\left(125 \mathrm{MHz}, \mathrm{DMSO}-d_{6}\right): \delta_{\mathrm{C}} 20.8,65.3,115.1,118.0,121.7$, $124.4,126.3,127.8,128.3,129.0,129.3,129.4,129.9,132.4,133.4,134.5,135.0,136.8,141.1,143.2,146.9$, $154.8,161.2$.

Ethyl 2-(2-chlorophenyl)-1-phenyl-1H-imidazole-5-carboxylate (8a). Brown solid; mp: $158-160{ }^{\circ} \mathrm{C} . ~ I R ~ \mathrm{v} / \mathrm{cm}^{-1}$ $(\mathrm{KBr}): 1728(\mathrm{C}=\mathrm{O}), 1598(\mathrm{C}=\mathrm{N}) \mathrm{cm}^{-1} .{ }^{1} \mathrm{H}$ NMR $\left(500 \mathrm{MHz}, \mathrm{DMSO}-d_{6}\right): \delta_{\mathrm{H}} 1.32\left(\mathrm{t}, J 5.6,3 \mathrm{H}, \mathrm{CH}_{3}\right), 4.36(\mathrm{q}, \mathrm{J} 5.6,2 \mathrm{H}$, $\mathrm{CH}_{2}$ ), 7.23-7.48 (m, 7H, ArH), $7.59(\mathrm{~d}, J 8.5,2 \mathrm{H}, \mathrm{ArH}), 8.33(\mathrm{~s}, 1 \mathrm{H}, \mathrm{CH}) .{ }^{13} \mathrm{C} N M R\left(125 \mathrm{MHz}, \mathrm{DMSO}-d_{6}\right): \delta_{\mathrm{c}} 14.3$, 59.8, 124.8, 127.2, 127.8, 128.9, 129.2, 129.4, 131.6, 132.5, 132.9, 133.1, 134.5, 136.4, 144.7, 162.1. Anal. Calcd for $\mathrm{C}_{18} \mathrm{H}_{15} \mathrm{ClN}_{2} \mathrm{O}_{2}$ (326.78): C, 66.16; $\mathrm{H}, 4.63 ; \mathrm{N}, 8.57$. Found: C, 66.47; H, 4.71; N, 8.54.

Ethyl 1-(4-bromophenyl)-2-(2-chlorophenyl)-1H-imidazole-5-carboxylate (8b). Brown solid; mp: $151-153{ }^{\circ} \mathrm{C}$. IR v/cm ${ }^{-1}(\mathrm{KBr}): 1727(\mathrm{C}=\mathrm{O}), 1549(\mathrm{C}=\mathrm{N}) \mathrm{cm}^{-1} .{ }^{1} \mathrm{H}$ NMR $\left(500 \mathrm{MHz}, \mathrm{DMSO}-d_{6}\right): \delta_{\mathrm{H}} 1.32\left(\mathrm{t}, J 5.6,3 \mathrm{H}, \mathrm{CH}_{3}\right), 4.28(\mathrm{q}$, J 5.6, 2H, $\mathrm{CH}_{2}$ ), $7.22(\mathrm{~d}, J$ 8.5, 2H, ArH), 7.45-7.49 (m, 3H, ArH), $7.53(\mathrm{~d}, J$ 8.5, 2H, ArH), $7.65(\mathrm{~d}, J$ 9.0, 1H, ArH), $8.38(\mathrm{~s}, 1 \mathrm{H}, \mathrm{CH}) .{ }^{13} \mathrm{C}$ NMR $\left(125 \mathrm{MHz}\right.$, DMSO- $\left.d_{6}\right): \delta_{\mathrm{C}} 14.7,60.3,121.9,127.3,127.4,127.5,127.7,128.3,129.6$, 129.8, 132.2, 132.7, 133.5, 136.2, 145.1, 162.4. $\mathrm{C}_{18} \mathrm{H}_{14} \mathrm{BrClN}_{2} \mathrm{O}_{2}$ (405.68): C, 53.29; H, 3.48; N, 6.91. Found: C, $53.37 ; \mathrm{H}, 3.52 ; \mathrm{N}, 6.83$.

Ethyl 1-(4-chlorophenyl)-2-phenyl-1H-imidazole-5-carboxylate (8c). Brown solid; mp: $163-165{ }^{\circ} \mathrm{C} . \mathrm{IR} \mathrm{v}^{\mathrm{v}} / \mathrm{cm}^{-1}$ $(\mathrm{KBr}): 1724(\mathrm{C}=\mathrm{O}), 1563(\mathrm{C}=\mathrm{N}) \mathrm{cm}^{-1} .{ }^{1} \mathrm{H} N M R\left(500 \mathrm{MHz}, \mathrm{DMSO}-d_{6}\right): \delta_{\mathrm{H}} 1.30\left(\mathrm{t}, J 5.8,3 \mathrm{H}, \mathrm{CH}_{3}\right), 4.27(\mathrm{q}, J 5.8,2 \mathrm{H}$, $\left.\mathrm{CH}_{2}\right), 7.21-7.44(\mathrm{~m}, 7 \mathrm{H}, \mathrm{ArH}), 7.63(\mathrm{~d}, J 8.0,2 \mathrm{H}, \mathrm{ArH}), 8.31(\mathrm{~s}, 1 \mathrm{H}, \mathrm{CH}) .{ }^{13} \mathrm{C} \mathrm{NMR}\left(125 \mathrm{MHz}, \mathrm{DMSO}-d_{6}\right): \delta_{\mathrm{C}} 15.3$, $59.73,122.6,126.3,127.8,128.1,129.4,130.8,132.9,133.2,134.5,138.7,146.6,161.7 . \mathrm{C}_{18} \mathrm{H}_{15} \mathrm{ClN}_{2} \mathrm{O}_{2}$ (326.78): C, 66.16; H, 4.63; N, 8.57. Found: C, 66.32; H, 4.69; N, 8.48.

Ethyl 2-phenyl-1-(p-tolyl)-1H-imidazole-5-carboxylate (8d). Brown solid; mp: $148-150{ }^{\circ} \mathrm{C} . \mathrm{IR} \mathrm{v} / \mathrm{cm}^{-1}(\mathrm{KBr})$ : $1739(\mathrm{C}=\mathrm{O}), 1592(\mathrm{C}=\mathrm{N}) \mathrm{cm}^{-1} .{ }^{1} \mathrm{H}$ NMR $\left(500 \mathrm{MHz}, \mathrm{DMSO}-d_{6}\right): \delta_{\mathrm{H}} 1.29\left(\mathrm{t}, J 5.6,3 \mathrm{H}, \mathrm{CH}_{3}\right), 2.34\left(\mathrm{~s}, 3 \mathrm{H}, \mathrm{CH}_{3}\right), 4.27$ (q, J 5.6, 2H, CH 2 ), $7.22\left(\mathrm{~d}, J\right.$ 8.5, 2H, ArH), 7.27 (d, J 8.5, 2H, ArH), 7.32-7.37 (m, 5H, ArH), $8.11(\mathrm{~s}, 1 \mathrm{H}, \mathrm{CH}) .{ }^{13} \mathrm{C}$ NMR $\left(125 \mathrm{MHz}\right.$, DMSO-d $\left.d_{6}\right): \delta_{c} 14.9,21.1,60.8,122.7,125.6,127.8,128.1,129.2,130.5,132.3,132.8,134.4$, 135.2, 145.8, 160.9. $\mathrm{C}_{19} \mathrm{H}_{18} \mathrm{~N}_{2} \mathrm{O}_{2}$ (306.37): C, 74.49; $\mathrm{H}, 5.92 ; \mathrm{N}, 9.14$. Found: $\mathrm{C}, 74.22 ; \mathrm{H}, 5.83 ; \mathrm{N}, 9.10$.

\section{Supplementary Material}

All the spectral IR, ${ }^{1} \mathrm{H} N M R$, and ${ }^{13} \mathrm{C}$ NMR data for compounds $\mathbf{6 a}-\mathbf{h}, \mathbf{8} \mathbf{a}-\mathbf{b}$ and $\mathbf{8 d}$ are provided in the Supplementary Material in the online version of the text.

\section{References}

1. Wen, S. Q.; Jeyakkumar, P.; Avula, S. R.; Zhang, L.; Zhou, C. H. Bioorg. Med. Chem. Lett. 2016, 26, 2768. https://doi.org/10.1016/i.bmcl.2016.04.070

2. Li, Z. W.; Zhong, C. Y.; Wang, X. R.; Li, S. N.; Pan, C. Y.; Wang, X.; Sun, X. Y. Molecules 2020, $25,4293$. 
https://doi.org/10.3390/molecules25184293

3. Nikitina, P. A.; Bormotov, N. I.; Shishkina, L. N.; Tikhonov, A. Y.; Perevalov, V. P. Russ. Chem. Bull. 2019, 68, 634.

https://doi.org/10.1007/s11172-019-2467-6

4. Zhao, S.; Zhao, L.; Zhang, X.; Wei, P.; Wu, M.; Su, X.; Sun, B.; Zhao, D.; Cheng, M. Bioorg. Med. Chem. Lett. 2019, 29, 2448.

https://doi.org/10.1016/j.bmcl.2019.07.037

5. Ghorbani-Vaghei, R.; Izadkhah, V.; Mahmoodi, J.; Karamian, R.; Khoei, M. A. Monatsh. Chem. 2018, 149, 1447.

https://doi.org/10.1007/s00706-018-2167-1

6. Vazquez-Salazar, A.; Becerra, A.; Lazcano, A. PLoS One 2018, 13, e0196349.

https://doi.org/10.1371/journal.pone.0196349

7. Watanabe, M.; Kobayashi, T.; Ito, Y.; Fukuda, H.; Yamada, S.; Arisawa, M.; Shuto, S. Bioorg. Med. Chem. Lett. 2018, 28, 3630.

https://doi.org/10.1016/i.bmcl.2018.10.041

8. Gao, J.; Zhou, Z.; Guo, J.; Guo, Chem. Commun. 2017, 53, 6227. https://doi.org/10.1039/C7CC03056H

9. Abd El-Halim, H. F.; Nour El-Dien, F. A.; Mohamed, G. G.; Mohamed, N. A. J. Therm. Anal. Calorim. 2012, $109,883$.

https://doi.org/10.1007/s10973-011-1784-2

10. Song, H.; Shin, H. S. Acta Crystallogr. C 1998, 54, 1675.

https://doi.org/10.1107/S0108270198006386

11. Heel, R. C.; Brogden, R. N.; Speight, T. M.; Avery, G. S. Drugs 1978, 16, 177. https://doi.org/10.2165/00003495-197816030-00001

12. Moutevelis-Minakakis, P.; Gianni, M.; Stougiannou, H.; Zoumpoulakis, P.; Zoga, A.; Vlahakos, A. D.; Iliodromitis, E.; Mavromoustakos, T. Bioorg. Med. Chem. Lett. 2003, 13, 1737.

https://doi.org/10.1016/S0960-894X(03)00251-8

13. McClellan, K. J.; Balfour, J. A. Drugs 1998, 55, 713.

https://doi.org/10.2165/00003495-199855050-00011

14. Saladino, R.; Sponer, J. E.; Sponer, J.; Costanzo, G.; Pino, S.; Di Mauro, E. Life 2018, 8, 24. https://doi.org/10.3390/life8020024

15. Szabla, R.; Sponer, J. E.; Sponer, J.; Sobolewski, A. L. Gora, R. W. Phys. Chem. Chem. Phys. 2014, $16,17617$. https://doi.org/10.1039/C4CP02074J

16. Choe, J. C. Chem. Phys. Lett. 2018, 708, 71.

https://doi.org/10.1016/i.cplett.2018.08.004

17. Leutou, A. S.; Yang, I.; Kang, H.; Seo, E. K.; Nam, S. J. Fenical, W. J. Nat. Prod. 2015, 78, 2846. https://doi.org/10.1021/acs.jnatprod.5b00746

18. Bennett Jr, L. L.; Baker, H. T. J. Am. Chem. Soc. 1957, 79, 2188. https://doi.org/10.1021/ja01566a044

19. Perdan-Pirkmajer, K.; Pirkmajer, S.; Thevis, M.; Thomas, A.; Praprotnik, S.; Hočevar, A.; Rotar, Ž.; Gašperšič, N.; Sodin-Šemrl, S.; Žibert, J.; Omersel, J.; Chibalin, A. V.; Tomšič, M.; Ambrožič, A. Scand. J. Rheumatol. 2016, 45, 347.

https://doi.org/10.3109/03009742.2015.1105290 
20. Francini, C. M.; Fallacara, A. L.; Artusi, R.; Mennuni, L.; Calgani, A.; Angelucci, A.; Schenone, S.; Botta, M. ChemMedChem 2015, 10, 2027.

https://doi.org/10.1002/cmdc.201500428

21. Francini, C. M.; Musumeci, F.; Fallacara, A. L.; Botta, L.; Molinari, A.; Artusi, R.; Mennuni, L.; Angelucci, A.; Schenone, S. Molecules 2018, 23, 2369.

https://doi.org/10.3390/molecules23092369

22. Helal, C. J.; Kang, Z.; Lucas, J. C.; Gant, T.; Ahlijanian, M. K.; Schachter, J. B.; Richter, K. E.; Cook, J. M.; Menniti, F. S.; Kelly, K.; Mente, S. Bioorg. Med. Chem. Lett. 2009, 19, 5703.

https://doi.org/10.1016/j.bmcl.2009.08.019

23. Karami, B.; Eskandari, K.; Farahi, M. Barmas, A. J. Chin. Chem. Soc. 2012, 59, 473. https://doi.org/10.1002/jccs.201100555

24. Vikrant, K.; Ritu, M.; Neha, S. Res. J. Chem. Sci. 2012, 2, 18.

25. Jayram, J.; Jeena, V. Green Chem. 2017, 19, 5841. https://doi.org/10.1039/C7GC02484C

26. Ahooie, T. S.; Azizi, N.; Yavari, I.; Hashemi, M. M. J. Iran. Chem. Soc. 2018, 15, 855. https://doi.org/10.1007/s13738-017-1284-9

27. Khandebharad, A. U.; Sarda, S. R.; Gill, C.; Agrawal, B. R. Org. Prep. Proced. Int. 2020, 52, 524. https://doi.org/10.1080/00304948.2020.1804773

28. Shaabani, A.; Rahmati, A. J. Mol. Catal. A: Chem. 2006, 249, 246. https://doi.org/10.1016/j.molcata.2006.01.006

29. Maleki, B.; Kahoo, G. E.; Tayebee, R. Org. Prep. Proced. Int. 2015, 47, 461. https://doi.org/10.1080/00304948.2015.1088757

30. Momahed Heravi, M.; Karimi, N.; Pooremami, S. Adv. J. Chem. A 2019, 2, 73. https://doi.org/10.29088/sami/AJCA.2019.2.7378

31. Hilal, D. A.; Hanoon, H. D. Res. Chem. Intermed. 2020, 46, 1521. https://doi.org/10.1007/s11164-019-04048-z

32. Wang, Y.; Shen, H.; Xie, Z. Synlett 2011, 7, 969. https://doi.org/10.1055/s-0030-1259713

33. Xu, W.; Wang, G.; Sun, N.; Liu, Y. Org. Lett. 2017, 19, 3307. https://doi.org/10.1021/acs.orglett.7b01469

34. Madhavachary, R.; Zarganes-Tzitzikas, T.; Patil, P.; Kurpiewska, K.; Kalinowska-Tluscik, J.; Domling, A. ACS Comb. Sci. 2018, 20, 192. https://doi.org/10.1021/acscombsci.7b00145

35. Chen, X.; Wang, Z.; Huang, H.; Deng, G. J. Adv. Synth. Catal. 2018, 360, 4017. https://doi.org/10.1002/adsc.201800765

36. Tian, Y.; Qin, M.; Yang, X.; Zhang, X.; Liu, Y.; Guo, X.; Chen, B. Tetrahedron 2019, 75, 2817. https://doi.org/10.1016/i.tet.2019.04.004

37. Xiong, J.; Wei, X.; Liu, Z. M.; Ding, M. W. J. Org. Chem. 2017, 82, 13735. https://doi.org/10.1021/acs.joc.7b02606

38. Mehrabi, H.; Alizadeh-Bami, F.; Ranjbar-Karimi, R. Tetrahedron Lett. 2018, 59, 1924. https://doi.org/10.1016/j.tetlet.2018.03.093

39. Alizadeh-Bami, F.; Salehzadeh, M.; Mehrabi, H.; Ranjbar-Karimi, R. ARKIVOC 2019, vi, 55. https://doi.org/10.24820/ark.5550190.p010.984 
40. Mehrabi, H.; Hajipour, M.; Rezazadeh-Jabalbarezi, F.; Alizadeh-Bami, F. J. Heterocycl. Chem. 2020, 57, 3361.

https://doi.org/10.1002/ihet.4053

41. Wang, Y.; Wang, H.; Peng, J.; Zhu, Q. Org. Lett. 2011, 13, 4604. https://doi.org/10.1021/ol201807n

42. Mehrabi, H.; Dastouri, F.; Asadi, S.; Alizadeh-Bami, F.; Ranjbar-Karimi, R. ARKIVOC 2020, vi, 114. https://doi.org/10.24820/ark.5550190.p011.151

43. Iravani, N.; Keshavarz, M.; Allah-Karampour, M. J. Sulfur Chem. 2018, 39, 414. https://doi.org/10.1080/17415993.2018.1441841

44. Yavari, I.; Hossaini, Z.; Shirgahi-Talari, F.; Seyfi, S. Synlett 2008, 11, 1631. https://doi.org/10.1055/s-2008-1077871

45. Yavari, I.; Seyfi, S.; Hossaini, Z.; Sabbaghan, M.; Shirgahi-Talari, F. Monatsh. Chem. 2008, 139, 1479. https://doi.org/10.1007/s00706-008-0953-x 